\title{
Observation of Sawtooth Redistribution of Nonthermal, Confined Alpha Particles in TFTR D-T Discharges
}

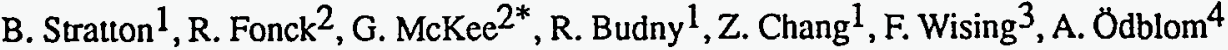 \\ 1Princeton Plasma Physics Laboratory, P. O. Box 451, Princeton, NJ 08534 \\ ${ }^{2}$ Department of Nuclear Engineering and Engineering Physics, \\ University of Wisconsin, Madison, WI 53706 \\ 3Plasma Fusion Center, Massachusetts Institute of Technology, Cambridge, MA 02139 \\ ${ }^{4}$ Institute for Electromagnetic Field Theory, Chalmers University of Technology, \\ S-41296 Gothenburg, Sweden
}

\begin{abstract}
Radial profiles of the density of confined alpha particles with energies in the $0.15-0.6 \mathrm{MeV}$ range are spectroscopically observed before and after a sawtooth crash in a TFTR D-T plasma. A large drop in the core alpha density is seen, indicating expulsion of alphas from the core to the plasma periphery. The measured changes in the alpha density profiles are consistent with predictions based on the Kolesnichenko sawtooth model, indicating that it may be used to reliably predict the effect of sawteeth on fusion-produced alphas.
\end{abstract}

*Present Address:

Oak Ridge National Laboratory

P.O. Box 2009

Bldg. 9201-2, MS-8072

Oak Ridge, TN 37831-8072

\section{DISCLAIMER}

This report was prepared as an account of work sponsored by an agency of the United States Government. Neither the United States Government nor any agency thereof, nor any of their employees, makes any warranty, express or implied, or assumes any legal liability or responsibility for the accuracy, completeness, or usefulness of any information, apparatus, product, or process disclosed, or represents that its use would not infringe privately owned rights. Reference herein to any specific commercial product, process, or service by trade name, trademark, manufacturer, or otherwise does not necessarily constitute or imply its endorsement, recommendation, or favoring by the United States Government or any agency thereof. The views and opinions of authors expressed herein do not necessarily state or reflect those of the United States Government or any agency thereof. 
Recent experiments on the Tokamak Fusion Test Reactor (TFTR) have allowed initial studies of confined alpha particle behavior in reactor-grade deuterium-tritium (D-T) tokamak plasmas. Neoclassical confinement and classical slowing-down of alphas in MHD-quiescent discharges have been observed in the 0.1-0.7 MeV energy range by the $\alpha$ CHERS diagnostic [1,2] and in the $0.5-2.0 \mathrm{MeV}$ range by the Pellet Charge Exchange diagnostic [3]. Both diagnostics have also seen evidence for redistribution of confined alphas following a sawtooth crash, but insufficient data were available in those early experiments to make detailed observations of the effect of sawteeth on the radial profiles of confined alphas. Measurements of lost alphas [4] during sawtceth in TFTR D-T discharges generally indicate that alphas are redistributed in the plasma, rather than ejected.

Understanding the effects of sawteeth on radial deposition of alpha heating power is crucial for design of future fusion reactors such as the International Thermonuclear Experimental Reactor (ITER). Kolesnichenko et al. [5] have shown that the alpha heating power in sawtoothing ITER discharges can be significantly decreased by the presence of sawteeth. The sawtooth model of Kolesnichenko et al. [6] has been successfully used to predict the observed redistribution of fast ions following a sawtooth crash in deuterium discharges on the Joint European Torus (JET) for a variety of fast ions: neutral beam-injected deuterium ions [7], fusion-produced protons and tritons [8], and large orbit width energetic ${ }^{3} \mathrm{He}$ ions produced by ICRF heating [9]. However, given the implications for ITER and other reactors, it is important to test this model against measurements of the effect of sawteeth on D-T alphas.

This Letter presents the first measurements which follow the alpha radial profile before and after a sawtooth crash in a single D-T tokamak plasma. A five-point radial profile of the alpha density in the $0.15-0.6 \mathrm{MeV}$ alpha energy region was measured by the $\alpha$-CHERS diagnostic [10]. Strong depletion of the core alpha density is seen following a sawtooth crash. The essential features of the measured profile after the crash are described well by the sawtooth model of Kolesnichenko et al. [6] using the pre-crash alpha density profile and q profiles similar to those measured [11] during sawtooth crashes on TFTR, indicating that this model may be used to reliably predict the effects of sawteeth on alpha heating in a reactor.

$\alpha$-CHERS [10] is a high-throughput optical system consisting of light collection optics fiber-optically coupled to three spectrometer/low-noise CCD camera systems. The energetic alpha signal is excited by charge exchange between alphas and heating beam deuterium atoms, and it appears as a weak wing on the long-wavelength side of the $\mathrm{He}^{+} 468.6$ $\mathrm{nm}$ line [1]. Five spatial channels are available, with sightlines intersecting three of the heating neutral beams in the toroidal midplane at radii spanning the region $\mathrm{r} / \mathrm{a}=0.05-0.6$ in the discharges studied here. Because the sightlines are nearly tangential to the flux surfaces where they intersect the beam trajectories, the radial resolution of each channel is 0.02-0.03 $\mathrm{m}$. Three spatial channels may be observed in a single discharge, making it necessary to combine data from two similar shots to obtain a complete five-point spatial profile. The cameras are read out at $0.05 \mathrm{~s}$ intervals, and the data were averaged over $0.2 \mathrm{~s}$ intervals to improve the signal-to-noise ratio in this experiment. The entire system is radiometrically calibrated, allowing absolute measurements of the alpha density to be made.

The experiment was performed in a standard TFTR D-T supershot [12] with a plasma current of $\mathrm{I}_{\mathrm{p}}=2.0 \mathrm{MA}$, toroidal magnetic field of $5.1 \mathrm{~T}$, and major and minor radii of $\mathrm{R}=2.52 \mathrm{~m}$ and $\mathrm{a}=0.87 \mathrm{~m}$, respectively. The time evolution of the injected neutral beam power, neutron source strength, and core electron temperature, $T_{e}(0)$, are shown in Fig. 1. Because the alpha slowing-down time was approximately $0.5 \mathrm{~s}$, five deuterium and three tritium beams were injected at energies of $100 \mathrm{keV}$ for a period of $1.3 \mathrm{~s}$ to establish a nearly steady-state alpha population in the intermediate energy range observed by $\alpha$-CHERS. The $95 \mathrm{keV}$ deuterium beams viewed by $\alpha$-CHERS were injected for an additional $0.7 \mathrm{~s}$, during which the alpha density radial profile was measured.

Sawteeth do not normally occur during the beam injection phase of supershots, so it was necessary to induce them by dropping the beam power $0.2 \mathrm{~s}$ after the end of the D-T beam phase, as seen in Fig. 1a. This caused the plasma $\beta$ 
to drop below the level required to suppress sawteeth and a sawtooth usually occurred within $0.25 \mathrm{~s}$ of the beam power drop, about halfway through the $\alpha$-CHERS observation period. As seen in Fig. 1b, there was only one sawtooth before the end of beam injection. By inducing the sawtooth in this way, the alpha density profile could be measured before and after the sawtooth crash. The alpha density profiles were obtained by combining data from two shots of this type. In the first shot the sawtooth crash occurred at $4.35 \mathrm{~s}$ and $\alpha$-CHERS measured the alpha density at the inner three radial points, while the crash occurred at $4.44 \mathrm{~s}$ in the second shot and $\alpha$-CHERS measured the alpha density at the outer two radii. Because there was no observed change in the alpha density in the outer two channels due to the sawtooth, the data from the two shots could be directly combined without taking into account the difference in the sawtooth crash times. The sawteeth were similar in both shots, with $\mathrm{T}_{\mathrm{e}}(0)=7.3 \mathrm{keV}$ and $\mathrm{n}_{\mathrm{e}}(0)=5.0 \times 10^{13} \mathrm{~cm}^{-3}$ before the sawtooth crash, and $\Delta \mathrm{T}_{\mathrm{e}}(0)=1.5 \mathrm{keV}$ and $\Delta \mathrm{n}_{\mathrm{e}}(0)=1.0 \times 10^{13} \mathrm{~cm}^{-3}$ at the sawtooth crash.

The alpha signal intensity is less than $1 \%$ of the bremsstrahlung background. The signal was extracted in the following way, described in detail by McKee et al. [2]. For each D-T shot, a D-T background shot was performed with the same total beam power and mixture of deuterium and tritium beams, but with the beams observed by $\alpha$-CHERS off during the 4.1-4.8 s period. This background shot has similar neutron production, electron density, and stored energy to the first (alpha signal) shot, and therefore has a similar bremsstrahlung background without the alpha signal. The edge impurity lines are fitted and removed from the spectra from both shots and the data are averaged over $0.8 \mathrm{~nm}$ spectral bins, corresponding to an alpha energy resolution of $0.03-0.09 \mathrm{MeV}$. The spectra are normalized to each other in the region of the spectrum corresponding to alpha energies of $0.7-1.0 \mathrm{MeV}$, where the alpha signal is too weak to be observable due to the falling charge exchange cross section, and the background spectrum is subtracted from the signal spectrum, resulting in the alpha signal.

The measured alpha spectra are modeled from TRANSP code [13] predictions of the alpha distribution function at each time and radial point in these specific discharges, beam atom densities calculated by a beam attenuation code [14], and cascade-corrected line excitation cross sections [15]. This procedure is described in detail in reference 2 . The model includes two sources of signal: direct alpha-beam atom charge exchange and charge exchange between alphas and halo thermal neutrals in the vicinity of the beam. The contribution to the signal of plume $\mathrm{He}^{+}$ions created by beam atom-alpha charge exchange and excited by clectron- and proton-collisions is negligible and is therefore not included [1].

For the purpose of comparison with the sawtonth model, it is desirable to deduce alpha densities from the measured spectra. To do this, the measured and predicted spectra and TRANSP alpha density at each time and radial point were integrated over the $0.15-0.6 \mathrm{MeV}$ alpha energy range to improve statistics, and the integrated TRANSP alpha density was then multiplied by the ratio of the measurcd alpha spectral signal to the predicted alpha signal. This process is appropriate because the shape of the measured alpha spectrum did not change within the noise on the signal during the sawtooth crash, and it is therefore just a direct normalization of the measured and predicted signals to obtain a measured alpha density. The TRANSP calculations assume a modified Kadomtsev [16] model of the sawtooth crash. The role of the TRANSP calculations in this analysis is to provide the relationship between the alpha energy distribution and the integrated alpha density. Detailed comparisons of $\alpha$-CHERS spectra and TRANSP predictions are discussed in reference 2.

The alpha density profiles measured at one time before the sawtooth crash $\left(t_{1}\right)$ and at two times after it $\left(t_{2}\right.$ and $t_{3}$ ) are shown in Fig. $2 a$; the integration time was $0.2 \mathrm{~s}$. The sawtooth crash occurred early in the $t_{2}$ time period. The error bars shown are based on the statistics of the signal, which is dominated by photon noise, and on systematic uncertainties resulting from the signal extraction process [2]. (The error bars on the outer two radial points are approximately the size of the plot symbols.) The major sources of uncerlainty in the predicted spectra used to deduce the alpha densities are uncertainties in the line-excitation charge exchange cross section and uncertainties in the beam stopping cross 
sections, which lead to uncertainties in the calculated beam densities [2]. The combined uncertainty in the deduced alpha densities from these sources ranges from $21 \%$ at $\mathrm{r} / \mathrm{a}=0.6$ to $32 \%$ at $\mathrm{r} / \mathrm{a}=0.05$. These uncertainties in the predicted spectra are not included in the error bars shown in Fig. 2a, because they affect only the absolute values of the alpha densities and do not contribute to uncertainties in relative changes in the alpha densities due to the sawtooth crash.

Several features of the effect of the sawtooth crash on the alpha density are clearly seen in Fig. 2a. There is a sharp drop in the core alpha density following the sawtooth crash, followed by partial recovery approximately $0.2 \mathrm{~s}$ later. Full recovery of the alpha signal is not expected because the suurce of $3.5 \mathrm{MeV}$ alphas dropped strongly at the end of $\mathrm{D}$ $T$ beam injection, well before the sawtooth crash. Electron cyclotron emission (ECE) measurements of the $T_{e}$ profile as a function of time yield an inversion radius of $r / a=0.25$ during this sawtooth crash. This is consistent with the point at which the curves $t_{1}$ and $t_{2}$ in Fig. $2 a$ cross. There is a small increase in the alpha density at $r / a=0.3$, which is just outside the inversion radius, although it is comparable to the size of the error bars. A large increase is not expected due to the long integration time of the measurements, since particles from the volume inside the inversion radius are displaced to a much larger volume outside it and and the effect of post-crash radial diffusion is significant, as discussed below. There is no significant change in the alpha density at the two outer-most radii. The lost-alpha detectors [4] showed no expulsion of alphas from the plasma during this sawtooth crash, indicating internal redistribution only.

The observed changes in the alpha density profiles shown in Fig. 2a were simulated with the sawtooth model of Kolesnichenko et al. [6]. This model is based on a Kadomtsev-like [16] rigid shift of the core plasma due to a $m=n=1$ kink instability combined with a quasi-interchange influx of external plasma to the core, as in the Wesson model [17]. This is expressed through conservation equations for particles, energy, and magnetic flux, and, unlike the Kadomtsev model, it allows $\mathrm{q}(0)<1$ following the crash due to the existence of two reconnection layers. The application of this model to the behavior of fast ions during a sawtooth crash is described in detail by Anderson et al. [7].

The post-crash alpha density profile is predicted by a transformation of the measured pre-crash profile based on input q profiles before and after the sawtooth crash. Measurements of the q profile were not available in these discharges, so q profiles similar to those measured before and after sawtooth crashes in other TFTR discharges were used [11]. The plasma redistribution is most sensitive to the values of the radius of the $q=1$ surface, $r_{S}$, and the mixing radius, $r_{\operatorname{mix}}$, and it is relatively insensitive to the detailed shape of the $q$ profile. The $q$ profile parameters used before the crash are $q(0)=0.87, r_{\mathcal{S}} / a=0.25$, and $r_{m i x} / a=\sqrt{2} \times r_{S} / a=0.35$, and after the crash: $q(0)=0.90$ and $r_{S} / a=0.24$. These values of $r_{\operatorname{mix}} / a$ and $\mathrm{r}_{\mathrm{S}} / \mathrm{a}$ are consistent with ECE $\mathrm{T}_{\mathrm{e}}$ profile measurements.

Figure $2 \mathrm{~b}$ shows the results of this model overlaid on the measured post-crash alpha density profile from the second time point in Fig. $2 \mathrm{a}, \mathrm{t}_{2}$. The four model curves correspond to different assumed values of the alpha radial diffusion coefficient, $\mathrm{D}_{\alpha}$, in the range 0 to $0.1 \mathrm{~m}^{2} / \mathrm{s}$. The radial diffusion is allowed to act for $0.2 \mathrm{~s}$, which corresponds to the signal integration time. It is clear that the observed large drop in the core alpha density is reproduced well by all values of $D_{\alpha}$ in this range, but that the relatively small increase in the alpha density outside the inversion radius is reproduced best by $D_{\alpha}=0.03 \mathrm{~m}^{2} / \mathrm{s}$. The neoclassical diffusion cocfficient for these discharges is estimated ${ }^{2}$ to be in the range 0.01 to $0.05 \mathrm{~m}^{2} / \mathrm{s}$, and $D_{\alpha}=0.03 \mathrm{~m}^{2} / \mathrm{s}$ is thus a reasonable value. It is also consistent with the upper limit on anomalous radial diffusion in addition to neoclassical diffusion measured by McKee et al. [2] to be in the range 0 to 0.03 $\mathrm{m}^{2} / \mathrm{s}$ in similar TFTR discharges. Finite orbit width effects are not included in this calculation. Typical orbit widths in the energy interval and radial region we are considering are 0.03-0.09 m; thus, there is also expected to be some smoothing of the sharp $D_{\alpha}=0$ post-crash profile shown in Fig. $2 b$, causing the peak to be less pronounced.

In summary, the radial profile of the density of confined alpha particles with energies in the range $0.15-0.6 \mathrm{MeV}$ has been measured before and after a sawtooth crash in a TFTR D-T plasma. A large drop in the core alpha density is seen. The sawtooth model of Kolesnichenko et al. [6] satisfactorily reproduces the essential features of the effect of the 
sawtooth crash on the measured alpha density profile, and can therefore be used with some confidence to predict the effect of sawteeth on alpha heating in future reactors such as ITER.

The authors would like to thank the TFTR group for supporting this experiment. In particular, J. Strachan provided useful advice on optimizing the discharges, $S$. Zweben provided lost alpha data, G. Taylor provided ECE $\mathrm{T}_{\mathrm{e}}$ data, and C. S. Chang performed neoclassical transport calculations. We would also like to thank R. Ashley, D. Cylinder, T. Thorson, and M. Vocaturo for hardware support, and T. Gibney, P. Roney, N. Schechtman, and H. Towner for software support. This work was supported by D. O. E. Contract No. DE-AC02-76CHO3073 and Grant No. DE-FG02-89ER53296, and by the Swedish Natural Science Research Council.

\section{REFERENCES}

[1] McKee, G., et al., Phys. Rev. Lett. 75 (1995) 649.

[2] McKee, G., et al., submitted to Nucl. Fusion (Dec. 1995).

[3] Fisher, R. K., et al., Phys. Rev. Lett. 75 (1995) 846.

[4] Zweben, S., et al., Phys. Plasmas 1 (1994) 1469.

[5] Kolesnichenko, Ya. I., Lutsenko, V. V., and Yakovenko, Yu. V., Fusion Technology 25

(1994) 302, and refs. therein.

[6] Kolesnichenko, Ya I., et al., Phys. Rev. Lett. 68 (1992) 3881.

[7] Anderson, D., et al., Nucl. Fusion 34 (1994) 217, and refs. therein.

[8] Anderson, D., et al., Plasma Phys. Control. Fusion 35 (1993) 733.

[9] Odblom, A., et al., Nucl. Fusion 35 (1995) 1571.

[10] McKee, G., et al., Rev. Sci. Instrum. 66 (1995) 643, and refs. therein.

[11] Levinton, F. M., et al., Phys. Fluids B 5 (1993) 2554.

[12] Strachan, J. D., et al., Phys. Rev. Lett. 72 (1994) 3526.

[13] Budny, R. V., et al., Nucl. Fusion 35 (1995) 1497.

[14] Janev, R. K., Boley, C. D., and Post, D. E., Nucl. Fusion 29 (1989) 2125.

[15] von Hellermann, M. G., et al., Plasma Phys. Controlled Fusion 33 (1991) 1805.

[16] Kadomtsev, B. B., Sov. J. Plasma Phys. (Engl. Trans.) 1 (1976) 389.

[17] Wesson, J. A., Plasma Phys. Control. Fusion 28 (1986) 243.

\section{FIGURE CAPTIONS}

FIG. 1. Plasma parameters for sawtooth measurements: a) neutral beam power, b) neutron rate, and c) $\mathrm{T}_{\mathrm{e}}(0)$.

FIG. 2. a) Measured alpha density profiles before sawtooth crash $\left(t_{1}\right)$ and at two times after sawtooth crash ( $t_{2}$ and $\left.t_{3}\right)$. b) Measured post-crash alpha density profile at time $l_{2}$ compared with model profiles assuming various values of $D_{\alpha}$. 

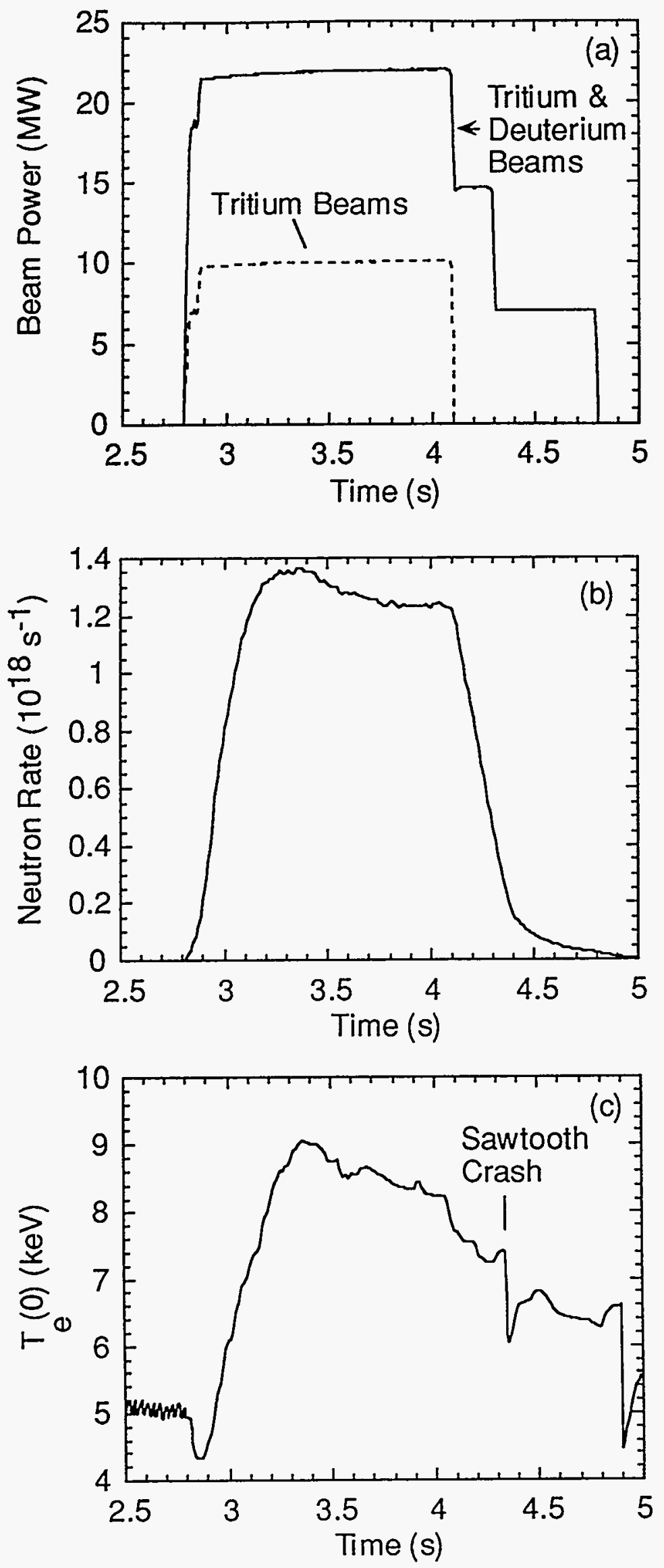

Figure 1 

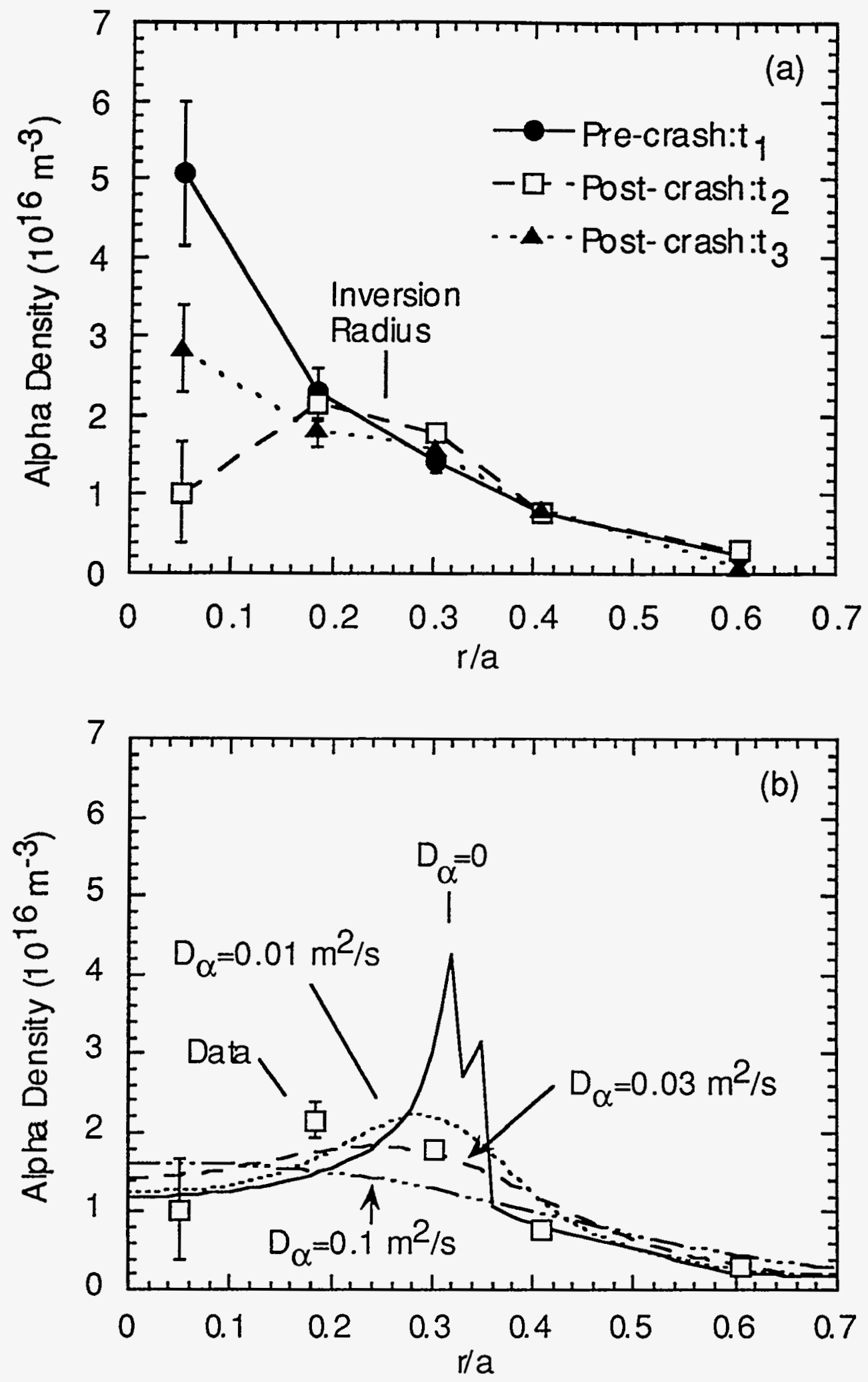

Figure 2 